\title{
Carbon based material for dye sensitized solar cells
}

\begin{abstract}
Dye sensitized solar cells (DSSC) is the third generation of solar cells that promise a simple procedure and low cost material which can provide electricity by converting the light energy into electrical energy. DSSC is a sandwich-liked structure that consists of conducting electrodes, sensitizer and electrolyte. In this research, Carbon Quantum Dots (CQDs) and Reduction Graphene Oxide (RGO) are used as organic sensitizer for DSSC and then it will be examined by scanning electron microscopy, ultraviolet visible spectroscopy, Raman spectroscopy, and solar simulator to observe the optical properties, optical properties, quality and efficiency of DSSC respectively. The results show that RGO produce a higher efficiency compared to CQD as sensitizer in DSSCs.
\end{abstract}

Keyword: Dye sensitized solar cells; Photosensitizer; Dye; Power conversion efficiency 